\title{
Aplicación de las Sustituciones de Descartes y Cardano para la Solución de Polinomios de Tercer y Cuarto Orden
}

\author{
Application of the Substitutions of Descartes and \\ Cardano for the Solution of Polynomials of the Third \\ and Fourth Order
}

\author{
J. A. Ramírez-Monares ${ }^{1}$ \\ ${ }^{1}$ Universidad Autónoma de Ciudad Juárez.
}

\section{RESUMEN}

El objetivo del presente trabajo es representar en un diagrama de Campbell el cambio en las frecuencias naturales con respecto a la velocidad de rotación $\Omega$ de un rotor con 2 grados de libertad. Dichas frecuencias naturales son la parte imaginaria de las raíces del polinomio de cuarto orden. Estas se obtienen mediante las sustituciones de Descartes y Cardano que reducen el orden de los polinomios con coeficientes escalares. Con las sustituciones de Cardano, el término cúbico en un polinomio general de cuarto orden es eliminado y el polinomio es reducido posteriormente a uno de orden 3. Con las sustituciones de Descartes, el término cuadrático del polinomio general de orden 3 es eliminado y posteriormente el polinomio es reducido a uno de grado 2, el cual es resuelto finalmente por la bien conocida fórmula general. En el diagrama de Campbell se aprecia el cambio en las raíces con respecto a la velocidad de rotación. Los métodos aquí presentados para la obtención de las raíces no son un análisis de estabilidad del sistema mecánico, sin embargo, simplifican el esfuerzo de cálculo para la obtención de las mismas.

PALABRAS CLAVE: Sustituciones de Cardano; sustituciones de Descartes; solución de polinomios; orden de polinomios.

\section{ABSTRACT}

The aim of this paper is to represent in a Campbell diagram the change in natural frequencies with respect to the rotation speed $\Omega$ of a rotor with 2 degrees of freedom. These natural frequencies are the imaginary part of the roots of the fourth order polynomial. These are obtained through the substitutions of Descartes and Cardano that reduce the order of the polynomials with scalar coefficients. For a general form of fourth-order polynomial, by replacing Cardan the cubic term is eliminated and reduced to a general polynomial of order 3. With the substitutions of Descartes, the quadratic terms of the polynomial of degree 3 is first eliminated, then reduces to a polynomial of degree two, which is finally solved by the well-known general formula. The Campbell diagram shows the change in roots with respect to the speed of rotation. The methods presented here for obtaining the roots are not an analysis of the stability of the mechanical system, however, they simplify the calculation effort to obtain them.

KEYWORDS: Cardano substitutions; Descartes substitutions; polynomials solutions; polynomial order.

Correspondencia:

DESTINATARIO: José Alfredo Ramírez Monares INSTITUCIÓN: Universidad Autónoma de Ciudad Juárez / División Multidisciplinaria de Ciudad Universitaria. DIRECCIÓN: Av. José de Jesús Macías Delgado núm. 18100, C. P. 32579, Ciudad Juárez, Chih. México. CORREO ELECTRÓNICO: jose.ramirez@uacj.mx
Fecha de recepción:

19 de junio de 2019

Fecha de aceptación:

3 de septiembre de 2019 


\section{INTRODUCCIÓN}

Existen diversas publicaciones en lo que se refiere a los métodos analíticos de solución de polinomios de cuarto orden con coeficientes reales. Alcalá ${ }^{[1]}$ presenta un desarrollo detallado de las sustituciones hechas a un polinomio de cuarto grado que no está en su forma general, es decir, carece de los términos cúbico y cuadrático. Esto, a diferencia de ${ }^{[2]}$, donde sí se presenta el polinomio general de cuarto grado en su forma canónica, aunque solo se reduce de orden mediante la sustitución de Descartes. Fathi et al. ${ }^{[3]}$ proponen lo que denominan "un método simple para resolver las ecuaciones cuárticas", que aquí es utilizado en las sustituciones 1 y 2. En ${ }^{[4]}$ se muestran diversos ejemplos de polinomios resueltos con las sustituciones aquí presentadas.

La ventaja de utilizar estos métodos de solución en problemas de rotodinámica radica en que requiere un menor esfuerzo de cómputo en comparación con la solución del problema del eigenvalor. Es aquí donde radica la importancia del presente método, ya que puede simplificar el esfuerzo de cálculo requerido al determinar las raíces de forma iterativa en un problema de optimización de vibraciones mecánicas.

\section{METODOLOGÍA}

Aquí se muestra una aplicación de las sustituciones de Cardano ${ }^{[1]}$ para resolver polinomios de cuarto orden, de acuerdo con ${ }^{[2]}$ en su forma canónica:

$$
a^{\prime} x^{4}+b^{\prime} x^{3}+c^{\prime} x^{2}+d^{\prime} x+e^{\prime}=0
$$

reduciéndolos de orden mediante sustituciones, como se muestra en la Figura 1.

\section{A. Sustitución 1 del Polinomio de Cuarto Orden}

El método de Descartes presentado en ${ }^{[1]} \mathrm{y}^{[3]}$, denominado transformación de Tschirnhaus en ${ }^{[5]}$, consiste en hacer la sustitución:

$$
x=z-\frac{b^{\prime}}{4}
$$

en el polinomio de cuarto orden. De esta forma, el nuevo polinomio en términos de $z$ está dado por:

$$
z^{4}+p z^{2}+q z+r=0
$$

donde los nuevos coeficientes están definidos por:

$$
\begin{gathered}
p=c^{\prime}-\frac{3 b^{\prime 2}}{8} \\
q=d^{\prime}-\frac{b^{\prime} c^{\prime}}{2}+\frac{b^{\prime 3}}{8} \\
r=e^{\prime}-\frac{b^{\prime} d^{\prime}}{4}+\frac{b^{\prime 2} c^{\prime}}{16}-\frac{3 b^{\prime 4}}{256}
\end{gathered}
$$

En esta sustitución desaparece el término cúbico del polinomio de cuarto orden.

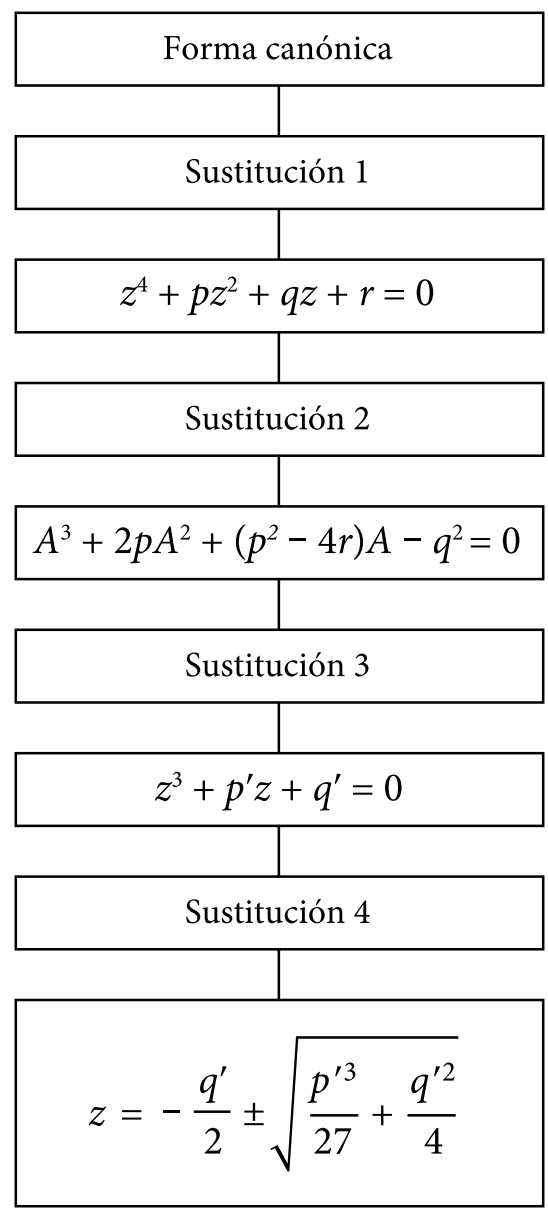

Figura 1. Metodología para la búsqueda de las raíces.

\section{B. Sustitución 2 del Polinomio de Cuarto Orden}

El método de Descartes presentado en ${ }^{[6]}$ consiste en utilizar un polinomio de la forma: 


$$
\left(z^{2}+\alpha z+\beta\right)\left(z^{2}-\alpha z+\gamma\right)=0
$$

cuya solución en términos de $z$ está dada por:

$$
\begin{aligned}
& z_{1,2}=\frac{\alpha \pm \sqrt{\alpha^{2}-4 \beta}}{2} \\
& z_{3,4}=\frac{\alpha \pm \sqrt{\alpha^{2}-4 \gamma}}{2}
\end{aligned}
$$

para solucionar el polinomio mostrado en (1). De acuerdo con Alcalá ${ }^{[1]}$ y Stewart ${ }^{[5]}$, el método de Descartes consiste en igualarlo al polinomio mostrado en (7), obteniéndose:

$$
\begin{gathered}
z^{4}+\left(\gamma+\beta-\alpha^{2}\right) z^{2}+\alpha(\gamma-\beta) z+\beta \gamma \\
=z^{4}+p z^{2}+q z+r
\end{gathered}
$$

lo cual conduce al sistema:

$$
\begin{gathered}
\beta+\gamma-\alpha^{2}=p \\
\alpha(\gamma-\beta)=q \\
\beta \gamma=r
\end{gathered}
$$

De las ecuaciones (11) y (13) se obtienen:

$$
\begin{aligned}
& \beta=\frac{1}{2}\left(p+\alpha^{2}-\frac{q}{\alpha}\right) \\
& \gamma=\frac{1}{2}\left(p+\alpha^{2}+\frac{q}{\alpha}\right)
\end{aligned}
$$

La solución del sistema (11) y (13) conduce al polinomio siguiente:

$$
\alpha^{6}+2 p \alpha^{4}+\left(p^{2}-4 r\right) \alpha^{2}-q^{2}=0
$$

Al hacer uso de la sustitución:

$$
\alpha^{2}=A
$$

en (16), finalmente se tiene:

$$
A^{3}+2 p A^{2}+\left(p^{2}-4 r\right) A-q^{2}=0
$$

\section{Sustitución 3 del Polinomio de Tercer Orden}

La ecuación (18) es un polinomio de tercer orden, la cual es posible resolver mediante las sustituciones de
Cardano presentadas en ${ }^{[4]},{ }^{[6]} \mathrm{y}^{[7]}$, también denominada transformación de Tschirnhaus en ${ }^{[5]}$. Este procedimiento es para transformar polinomios de la forma:

$$
A^{3}+a A^{2}+b A+c=0
$$

en nuevos polinomios sin el término cuadrático en términos de otra variable. Esto se logra con las sustituciones:

$$
\begin{gathered}
A=z-\frac{a}{3} \\
A^{2}=z^{2}-\frac{2}{3} a z+\frac{\alpha^{2}}{9} \\
A^{3}=z^{3}-a z^{2}+\frac{a^{2}}{3} z-\frac{a^{3}}{27}
\end{gathered}
$$

lo cual resultan en:

$$
z^{3}+\left(b-\frac{a^{2}}{3}\right) z+\frac{2}{27} a^{3}-\frac{a b}{3}+c=0
$$

Esta es una ecuación de la forma:

$$
z^{3}+p^{\prime} z+q^{\prime}=0
$$

donde:

$$
\begin{gathered}
p^{\prime}=b-\frac{a^{2}}{3} \\
q^{\prime}=\frac{2}{27} a^{3}-\frac{a b}{3}+c
\end{gathered}
$$

\section{Sustitución 4 del Polinomio de Tercer Orden}

Para dar solución a un polinomio de tercer grado como el que se muestra en la ecuación (24), se requiere hacer uso de las sustituciones:

$$
\begin{gathered}
z=(u+v) \\
z^{3}=u^{3}+v^{3}+3 u v(u+v)
\end{gathered}
$$

Al igualar los valores de $z^{3}$ en las ecuaciones (24) y (28), se obtienen:

$$
u^{3}+v^{3}+3 u v z=-p^{\prime} z-q^{\prime}
$$




$$
z^{2}+q^{\prime} z-\frac{q^{\prime 3}}{27}=0
$$

Para completar el binomio cuadrado perfecto en la ecuación (30), se tienen:

$$
\begin{gathered}
z^{2}+q^{\prime} z+\frac{q^{\prime 2}}{4}=\frac{p^{\prime 3}}{27}+\frac{q^{\prime 2}}{4} \\
\left(z+\frac{q^{\prime}}{2}\right)^{2}=\frac{p^{\prime 3}}{27}+\frac{q^{\prime 2}}{4}
\end{gathered}
$$

Despejando a $z$ de (32), queda:

$$
z=\frac{q^{\prime}}{2} \pm \sqrt{\frac{p^{\prime 3}}{27}+\frac{q^{\prime 2}}{4}}
$$

La ecuación (33) es conocida como la fórmula de Cardano. Es importante analizar los posibles valores del extremo derecho de la ecuación (32). De acuerdo con Abramowitz y Stegun ${ }^{[8]}$, si el valor de estos términos es mayor que cero, entonces existe una raíz real y un par de raíces complejas conjugadas; si es igual a cero todas las raíces son reales y al menos dos de ellas son iguales; $\mathrm{y}$ si es menor que cero todas las raíces son reales.

\section{RESULTADOS}

\section{A. Descripción del Modelo Mecánico}

El siguiente caso es presentado por Lalanne y Ferraris ${ }^{[9]}$. Considérese un sistema de referencia inercial $R_{0}$ $(X, Y, Z)$ : el eje del rotor está a lo largo del eje $Y$ y una velocidad de rotación constante $\Omega$, lo cual se muestra en la Figura 2. Solo se usa un grado de libertad para los desplazamientos en las direcciones X y Z. El rotor se considera simplemente apoyado en ambos extremos y está constituido por lo siguiente:

- una flecha simétrica de longitud $L$;

- un disco simétrico con una masa $m$ situado en $y=l_{1}$;

- un cojinete hidrodinámico con rigideces $k_{\mathrm{xx}}, k_{\mathrm{zz}}$, rigideces cruzadas $k_{\mathrm{xz}}, k_{\mathrm{zx}} \mathrm{y}$ amortiguamiento $c$, situado en $y=l_{2}$.

Las expresiones para los desplazamientos en las direcciones $\mathrm{X}$ y Z son, respectivamente:

$$
\begin{aligned}
& u(y, t)=f(y) q_{1}(t)=f(y) \boldsymbol{q}_{1} \\
& w(y, t)=f(y) q_{2}(t)=f(y) \boldsymbol{q}_{2}
\end{aligned}
$$

donde $q_{1}$ y $q_{2}$ son coordenadas generalizadas independientes. Al elegir una función de desplazamiento, se tiene:

$$
f(y)=\operatorname{sen}\left(\frac{\pi y}{L}\right)
$$

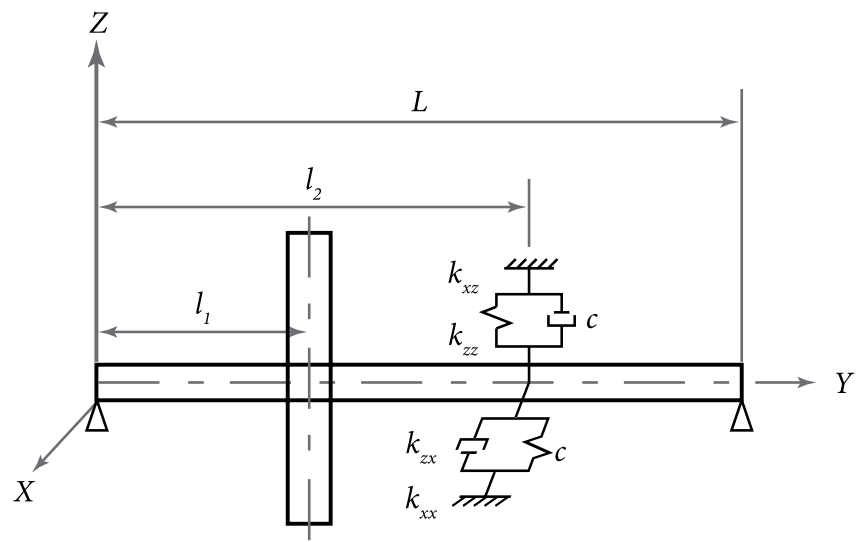

Figura 2. Modelo del rotor.

Y al aplicar las ecuaciones de Lagrange, considerando las energías cinética y potencial, se obtienen las ecuaciones de movimiento en estado estable del sistema mecánico:

$$
\begin{aligned}
& m \ddot{q}_{1}-a \Omega \dot{q}_{2}+c \dot{q}_{1}+k_{1} q_{1}+k_{12} q_{2}=0 \\
& m \ddot{q}_{2}+a \Omega \dot{q}_{1}+c \dot{q}_{2}+k_{2} q_{2}+k_{21} q_{1}=0
\end{aligned}
$$

donde $a$ es una constante relacionada con la geometría de la flecha. Las soluciones al movimiento en estado estable se pueden consideran como

$$
\begin{aligned}
& q_{1}=Q_{1} e^{r t} \\
& q_{2}=Q_{2} e^{r t}
\end{aligned}
$$

$\mathrm{Al}$ sustituir (37) en (36), resulta en el sistema de ecuaciones homogéneo:

$$
\begin{array}{r}
{\left[\begin{array}{rr}
m r^{2}+c r+k_{1} & -a \Omega r+k_{12} \\
a \Omega r+k_{21} & m r^{2}+c r+k_{2}
\end{array}\right]\left[\begin{array}{l}
Q_{1} \\
Q_{2}
\end{array}\right]} \\
=\left[\begin{array}{l}
0 \\
0
\end{array}\right]
\end{array}
$$


cuya solución trivial no es de interés. Las soluciones no triviales están asociadas con los valores que convierten en cero el determinante de la matriz.

La expansión del determinante de dicha matriz resulta en el polinomio característico:

$$
\begin{array}{r}
r^{4}+\frac{2 c}{m} r^{3}+\left(\frac{k_{1}}{m}+\frac{k_{2}}{m}+\frac{c^{2}}{m^{2}}+\frac{a^{2} \Omega^{2}}{m^{2}}\right) r^{2} \\
+\left[\frac{c k_{1}}{m^{2}}+\frac{c k_{2}}{m^{2}}+\frac{a}{m^{2}}\left(k_{21}-k_{12}\right) \Omega\right] r \\
+\frac{k_{1} k_{2}-k_{12} k_{21}}{m^{2}}=0
\end{array}
$$

cuyas raíces son las frecuencias naturales del sistema mecánico.

\section{B. Valores Numéricos}

En esta sección se asignan los valores numéricos $k_{x x}=$ $2 \times 10^{5} \mathrm{~N} / \mathrm{m}, k_{z z}=5 \times 10^{5} \mathrm{~N} / \mathrm{m}, k_{x z}=-k_{z x}=4 \times 10^{4} \mathrm{~N} / \mathrm{m}$, $c_{x x}=c_{z z}=10^{2} \mathrm{~N} / \mathrm{m} / \mathrm{s}$ en el polinomio característico (39) y se emplean las sustituciones de Descartes y Cardano para representar en un diagrama de Campbell el cambio en las frecuencias naturales con respecto a la frecuencia de rotación $\Omega(\mathrm{rad} / \mathrm{s})$ del rotor con 2 grados de libertad. El polinomio característico queda:

$$
\begin{array}{r}
r^{4}+10.49 r^{3}+\left(2.04 \times 10^{5}+4.034 \times 10^{-2} \Omega^{2}\right) r^{2} \\
+(1.07-843 \Omega) r+1.034 \times 10^{10}=0
\end{array}
$$

Este polinomio está en la forma de la ecuación (1), de donde se identifican los coeficientes:

$$
\begin{aligned}
& a^{\prime}=1 \\
& b^{\prime}=10.49 \\
& c^{\prime}=204000+0.04034 \Omega^{2} \\
& d^{\prime}=1.07-843 \Omega \\
& e^{\prime}=1.034 \times 10^{10}
\end{aligned}
$$

y los nuevos coeficientes definidos por las ecuaciones (4) a (6):

$$
\begin{aligned}
& p=203958.73+0.04034 \Omega^{2} \\
& q=-1.06 \times 10^{6}-843 \Omega-0.211 \Omega^{2} \\
& r=1.034 \times 10^{10}+2210.767 \Omega+0.2274 \Omega
\end{aligned}
$$

Utilizando las sustituciones mencionadas en las secciones anteriores para la reducción de orden de los polinomios, se obtienen las raíces del polinomio (40). En la Figura 3 se muestra el diagrama de Campbell obtenido.

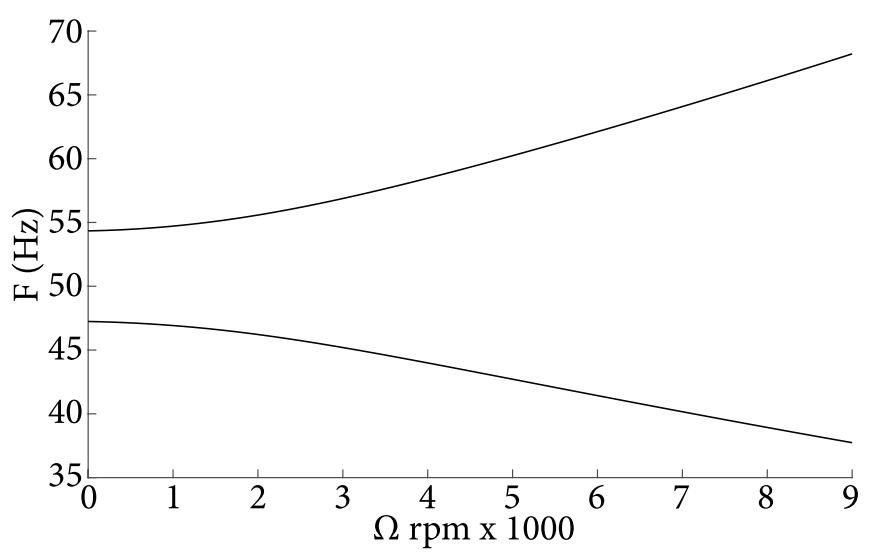

Figura 3. Diagrama de Campbell.

\section{CONCLUSIONES}

Se utilizaron las sustituciones de Cardano y Descartes para resolver un polinomio de cuarto grado cuyos coeficientes de los términos cuadrático y lineal dependen de la variable $\Omega$. También, se presentaron la parte imaginaria de las raíces del polinomio como función de $\Omega$ en el diagrama de Campbell. De esta forma queda demostrado el método aquí presentado para la solución del polinomio característico de cuarto orden, involucrando la velocidad de giro del rotor.

En su trabajo, Lalanne y Ferraris ${ }^{[9]}$ analizan el mismo polinomio con el criterio de Routh-Hurwitz, con el propósito de evaluar la estabilidad, la cual depende de los cambios de signo en la parte real de las raíces del polinomio característico. En el presente trabajo no se realiza un análisis de estabilidad, sino que se enfoca en el cálculo de las raíces en sí mismo. A pesar de esto, las sustituciones aquí presentadas pueden ser útiles para analizar probables cambios de signo en la parte real de las raíces del polinomio característico.

En cuanto al esfuerzo de cómputo para el cálculo de las raíces en el intervalo de valores de $\Omega$, el método aquí presentado requiere un tiempo de cálculo menor que la solución mediante el eigenvalor para cada valor de $\Omega$.

Las sustituciones aquí presentadas pueden ser línea de partida para problemas de optimización en el campo de vibraciones mecánicas, donde es frecuente encon- 
trar criterios de optimización basados en la separación de las frecuencias naturales, parte imaginaria de las raíces, dentro de un rango de velocidades $\Omega$.

Es importante mencionar también que los métodos aquí presentados para polinomios de tercer y cuarto orden restringen su aplicación a problemas de vibraciones mecánicas con máximo 2 grados de libertad, ya que un sistema vibratorio con más grados de libertad involucra un polinomio de mayor orden.

\section{REFERENCIAS}

[1] G. E. Alcalá, "Una Solución a la de Cuatro", rinconmatematico.com, 2006. [En línea]. Disponible en: http://rinconmatematico.com/foros/index.php/topic,38584.0.html.

[2] S. Janson, "Roots of Polynomials of Degrees 3 and 4", Cornell University, septiembre 13, 2010. [En línea]. Disponible en: https://arxiv.org/abs/1009.2373v1.

[3] A. Fathi, P. Mobadersany, y R. Fathi, "A Simple Method to Solve Quartic Equations”, Aust. J. Basic Appl. Sci., vol. 6, pp. 331-336, 2012.
[4] K. Ward, "Examples Using Cardano's Method to Solve Cubic Equations", Ken Ward's Mathematics Pages, 2019. [En línea]. Disponible en: https://trans4mind.com/ personal_development/mathematics/polynomials/cardanoMethodExamples.htm.

[5] I. Stewart, Galois theory, 3. ${ }^{\text {a }}$ ed., Florida: Chapman \& Hall/CRC, 2004.

[6] E. W. Weisstein, "Cubic Formula", WolframMathWorld, 2019. [En línea]. Disponible en: http://mathworld.wolfram.com/CubicFormula.html.

[7] A. Fathi y S. Nastaran, "A classic new method to solve quartic equations", Appl. Comput. Math., vol. 2, no. 2, pp. 24-27, 2013.

[8] M. Abramowitz y I. A. Stegun, Handbook of Mathematical Functions With Mathematical Functions with Formulas, Graphs, and Mathematical Tables, 10. ${ }^{a}$ ed., Washington: National Bureau of Standards Applied Mathematics Series 55, 1972.

[9] M. Lalanne y G. Ferraris, Rotordynamics Prediction in Engineering, 2. ${ }^{\text {a }}$ ed., Chichester: John Wiley and Sons, 1998. 